\title{
Measurement of human movement under metrological controlled conditions
}

\author{
Francesco Crenna, Giovanni Battista Rossi, Alice Palazzo \\ University of Genova, DIME/MEC, Via all'Opera Pia 15a, I-16145 GENOVA, Italy
}

\begin{abstract}
The study and modeling of human movement requires accurate measurement of forces and of kinematic quantities, with proper control and monitoring of measurement uncertainty. The metrological aspects of such experimentation are often overlooked in the biomechanical literature. In contrast, we present here a complete measurement system that combines a double force platform with vision and inertial sensors. Thanks to some degree of instrumental redundancy a cross-validation of different measuring channels is possible. Therefore the system permits the characterization of a person's movement, enabling, at the same time, the verification of the acquired experimental data. Details are provided about the system and about the measurement procedure, in order to allow the reproducibility of the experiment, if needed, in other laboratories. Then, as a test case, the experimental study of hopping is considered. Three male subjects performed the tests on several sessions. Experimental results are discussed, with special focus on kinematic quantities, and system performance is discussed, by properly accounting for uncertainty issues.
\end{abstract}

\section{Section: RESEARCH PAPER}

Keywords: complex measurement systems; kinematic measurements; biomechanics

Citation: Francesco Crenna, Giovanni Battista Rossi, Alice Palazzo, Measurement of human movement under metrological controlled conditions, Acta IMEKO, vol. 4, no. 4, article 10, December 2015, identifier: IMEKO-ACTA-04 (2015)-04-10

Editor: Paolo Carbone, University of Perugia, Italy

Received June 8, 2015; In final form December 3, 2015; Published December 2015

Copyright: (C) 2015 IMEKO. This is an open-access article distributed under the terms of the Creative Commons Attribution 3.0 License, which permits unrestricted use, distribution, and reproduction in any medium, provided the original author and source are credited

Funding: This research was partially funded through UNIGE research programs PRA 2013-2014.

Corresponding author: Francesco Crenna, e-mail: francesco.crenna@unige.it

\section{INTRODUCTION}

In biomechanical literature, generally speaking, the quality and reliability of measurement results is often taken as granted [1], [2]. Vendors of biomechanical instrumentation often refer to clinical studies, which give little metrological attention to reliability of measurement data [3]. Yet, a deeper investigation in motion generation and control, shows that measurement uncertainty plays a critical role [4]-[9]. For example, in the experimental study of human movement is it essential to be able to distinguish between inter-subjective variability, which is often the object of the investigation, and the dispersion due to measurement noise, also accounting for the indeterminacy due to measurement uncertainty related to systematic effects [4].

The study of human movement requires the measurement of both kinematics quantities, such as linear and angular displacement, velocity and acceleration of relevant body points and segments [5], and external forces that characterise the interaction of persons with the environment in which motion takes place. Internal driving forces produced by muscles may instead be indirectly measured starting from direct motion and external-forces measurement through the solution of an "inverse dynamic problem". In this latter case, the reduction of the uncertainty of initial direct measurement is particularly critical, due to uncertainty propagation effects.

Therefore, with the aim of contributing to improve the quality of measurements in experimental biomechanics, we present the design, realisation and testing of a complex measurement system, including some kinds of sensory redundancy, in order to ensure the reliability of the results and their metrological characterisation, in terms of uncertainty evaluation.

The system is based primarily on a video system operating with markers, together with inertial sensors and force platforms [7], [9]. Lower limbs kinematic may be obtained from two independent methods, so it is possible to provide verification by results comparison from the two methods [10]. The position 
of various points of interest in the sagittal plane can be obtained directly from video, or they can be also evaluated through the use of inertial data, measured on each body segment, and applied to the model of the limbs [10]-[12]. As test case we exploit the gesture of hopping, since it is frequently used in literature to describe inferior limbs behaviour [13]-[18].

The paper is organised as follows. In Section 2 we present a detailed description of the measuring system, outlining sensory redundancy and synchronisation issues. Then, after a brief presentation and motivation of the selected test case (in place hopping) (Section 3), in Section 4 we present the calibration and testing procedure, highlighting critical aspects, and providing enough details for the procedure to be reproduced and compared in other laboratories. Then, in Section 5 we present experimental results and provide an in depth discussion of uncertainty issues.

\section{MEASUREMENT SYSTEM}

A scheme of the general measurement setup is presented in Figure 1. The overall system consists of a combination of three subsystems, which were originally autonomous, and independent of each other: force platform, video and inertial measurement systems. In order to guarantee the same high reliability level of each subsystem as regards both data recording and timing, the overall setup synchronises them as communicating, but still autonomous systems. Such solution offers more reliability, at the cost of a more complex management of the set up. Let us now discuss how all this works.

\subsection{Markers and video system}

The video system is based on a black and white camera and a set of markers placed along the subject's leg in specific anatomical landmarks.

Two cameras have been considered and their performances have been evaluated. The first solution, more commonly used in our laboratory at present, consists in a BASLER camera, connected to a computer via a standard interface IEEE 1394 The alternative solution is based on a DALSA Falcon camera that has a higher resolution and a higher frame rate. The second option presents lower measurement uncertainty, mainly due to higher resolution, and a higher frame rate. A comparison of the two systems is reported in Table 1.

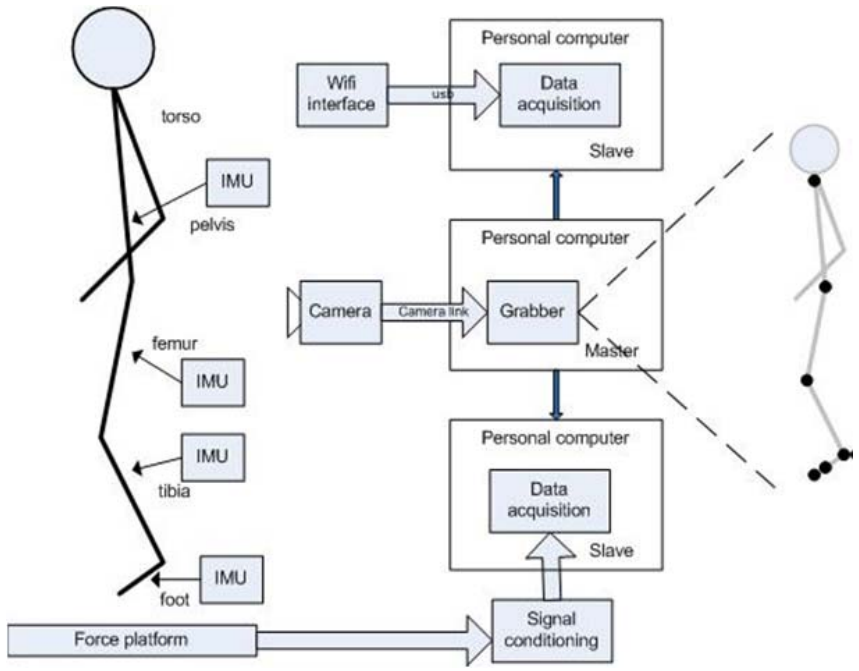

Figure 1. Schematic of the overall measurement system
A $12 \mathrm{~mm}$ focal length optics has been selected to have a complete view of the subject during hopping, from ground to head and shoulders. We have also considered the movement required in the test case. In this configuration the viewing angle in the vertical direction is about $30^{\circ}$.

The video system is completed with a set of active markers consisting of high intensity, $5 \mathrm{~mm}$ diameter, white led (2300 mcd @3.6 V and $20 \mathrm{~mA}$ ), which are patched over the subject's skin in the selected anatomical landmarks, facing the camera line of sight. In general a proper number of markers are used to identify specifically the movement of the most interesting landmarks, according to the biomechanical model in use. For example, if interested in lower limb movements, hip, knee and ankle movements are fundamental. Other markers might be placed on foot and/or along leg segments [6], [12], [13], [15]. Three markers are sufficient for the measurement of legsegments orientation and relative angles, at the ankle, and knee [10]. Anyway, the system is flexible and allows the processing of a variable number of markers, according to the test requirements.

Camera image acquisition parameters are settled to obtain a black image with white spots produced by the high intensity led, placed on anatomical landmarks. The video acquisition system saves an AVI file at $50 \mathrm{~Hz}$ frame rate, composed by bitmap raw images without any compression to maintain the original image resolution. The file is further processed to obtain marker positions in the image plane. Image processing is carried out off line: white spots are located in each image frame by frame and their positions are obtained, as their white level weighted centres. Therefore, even if the spot diameter on the image of a $5 \mathrm{~mm}$ led diameter is larger than 2 pixels, marker position is determined with a sub-pixel resolution. Position on image plane is then transferred on the sagittal plane considering sensitivity obtained through a previous calibration, under the hypothesis that subject's movements are in a plane parallel to the image plane. Such hypothesis has to be verified through experimental results.

\subsection{Inertial measurement system}

The subject under test is equipped also with a set of inertial sensors (Inertial Measurement Unit, IMU), model MTw by Xsens Technology. Each unit includes tri-axial accelerometer, gyroscope and magnetometer, and is wirelessly connected to a master base station. Some relevant technical features are reported in Table 2. IMU signals are internally pre-processed by the original Xsens software, yielding the orientation of each sensor in the three dimensional space.

Sensor boxes (of dimensions $34.5 \times 57.8 \times 14.5 \mathrm{~mm}(\mathrm{~W} \times \mathrm{L}$ $\times \mathrm{H})$ ) are placed on the subject's body. For the monitoring of lower limb movement, they are placed on foot, tibiae, thigh and usually on torso too, by elastic Velcro ${ }^{\circledR}$ strips with sensor

Table 1. Video systems comparison.

\begin{tabular}{ccc}
\hline & Basler A601f & Dalsa Falcon \\
\hline Resolution [pixel] & $640 \times 480$ & $1400 \times 1024$ \\
Pixel size $[\mu \mathrm{m}]$ & $9.9 \times 9.9$ & $7.4 \times 7.4$ \\
Sensor dimension $[\mathrm{mm}]$ & 12.7 & 12.7 \\
Max. frame rate $[\mathrm{Hz}]$ & 60 & 100 \\
\hline Data transmission & IEEE 1394 & Camera link \\
\hline Spatial resolution (at 1.2 m & & 1.34 \\
distance camera-subject) & 2.94 & \\
[mm/pixel] & &
\end{tabular}


Table 2. Inertial sensors characteristics

\begin{tabular}{lc}
\hline & Xsens MTw \\
\hline Dynamic range & $200 \mathrm{rad} / \mathrm{s}-160 \mathrm{~m} / \mathrm{s}^{2}$ \\
Static accuracy (roll-pitch) & $0.5 \mathrm{deg}$ \\
Static accuracy (yaw) & $1 \mathrm{deg}$ \\
Angular resolution & $0.05 \mathrm{deg}$ \\
Internal sampling rate & $1800 \mathrm{~Hz}$ \\
Bandwidth & $>120 \mathrm{~Hz}$ \\
Wireless sampling rate (up to 6 sensors) & $75 \mathrm{~Hz}$ \\
\hline
\end{tabular}

supports [12]. Each unit has a local coordinate reference system (Lcs) and the system measures its angular position with reference to a fixed world reference system (Wcs) to the master station. Each unit is previously reset in order to have both $\mathrm{Y}_{\mathrm{Lcs}}$ and $Z_{\mathrm{Lcs}}$ axes coincident with the $\mathrm{W}_{\mathrm{cs}}$ ones and oriented along the same direction. $Y_{W_{c s}}$ and $Z_{W c s}$ are oriented along subject's frontal direction and vertically [10], [11], [19]. Acceleration data are also available to the user for any further investigation. The inertial measurement system is autonomous and with four IMU sensors wirelessly connected to the control station, the system operates at $75 \mathrm{~Hz}$ sampling frequency.

\subsection{Force measuring system}

The force measuring system consists of two P6000D platforms by BTS Engineering with their own data acquisition and processing system; some technical characteristics are given in Table 3. Each platform is equipped with four tri-axial force sensors and is able to measure the overall forces exchanged by subject with ground during movement (Ground Reaction Force, GRF) and the corresponding Centre of Pressure, CoP. The CoP provides the position on the ground plane (or platform plane in this case) where the force transmission between the subject and the ground takes place. The position of the CoP, as compared with that of the Centre of Mass (CoM), is a key quantity for studying, e.g., the subject's vertical stabilisation [20]-[21].

Platforms are initialised before every test in order to take into account their zero deviation; zero drift has been verified on a time span much longer than a test session and it resulted to be negligible. Platforms operate at $1 \mathrm{kHz}$ sampling frequency. During tests it is possible to load each platform with one foot or with both feet, in order to study postural or force transversal asymmetries. The former situation results with $\mathrm{CoP}$ and force data relative to each leg, while the latter measures the complete subject's body without left-right leg distinction.

\subsection{Measurement system synchronisation}

The video acquisition is autonomous and assumes the role of master in the synchronisation of the overall measurement system. It sends to IMU control station and to the force platforms a start and stop trigger to control data acquisition.

Table 3. Force measurement system

\begin{tabular}{lc}
\hline & BTS P6000D \\
\hline X - Y sensor range & Up to $\pm 2000 \mathrm{~N}$ \\
Z sensor range & Up to $2000 \mathrm{~N}$ \\
Sensitivity/Resolution & 16 bit over selected range \\
Sensitivity deviation on plate surface & $<1.0 \%$ Full Scale Output \\
Hysteresis & $<0.2 \%$ Full Scale Output \\
\hline
\end{tabular}

The IMU control station, which performs at the lowest sampling rate among all the equipment, sends a trigger signal to the video master with the timing of each inertial measurement on rising edges, useful during off line processing to eventually resynchronise all measurements.

\section{A TEST CASE: HOPPING}

In order to validate the proposed measurement system, we have considered a "reference" gesture, hopping in place, for which many experimental results have been reported in the literature, therefore providing a sort of validation reference [13]-[18], [20], [22], [23]. This gesture is on the one hand informative, in that it provides a good example of lower limb movement, on the other it may be satisfactorily described in a single plane, which constitutes a noteworthy simplification in motion analysis that allowed us to focus on metrological aspects.

Experimentation repeatability and reproducibility has been enhanced by controlling subject's hopping rate through a metronome. In fact, in previous studies a preferred hopping rate was identified, which seems to be quite independent of subject's physical characteristics and may therefore be kept, without interfering excessively on subject's attitude [10], [20], [13], [17].

Currently available studies on hopping mainly consider the evaluation of the moments required, at the limbs joints, for performing the gesture. For example, they investigate the adjustment of the lower limbs behaviour according to the required performance [24]-[25], or due to the geometric nature of the ground, such as inclined or uneven, or to ground's dynamic nature, such as rigid or compliant [13], [18], [22]. On the other hand, a deep investigation of the displacement of the CoM and CoP seems to be missing [17]. In contrast, these are important biomechanical variables, for investigating the motion control required to maintain the (dynamic) equilibrium in a fairly constant position along the horizontal, anterior-posterior axis, whilst cyclically moving along the vertical axis. These latter quantities will be therefore addressed in some detail.

\section{EXPERIMENTAL PROCEDURE}

As a part of the present metrological approach, a detailed measurement procedure, involving both instrumental setup and subject training, has been developed, with a special attention to ensure the highest achievable reproducibility.

\subsection{Video system calibration}

The video system is set up to have the image plane parallel to subject's sagittal plane. Before every test session, the camera distance from subject is adjusted to obtain a whole body image starting from the lower edge of the force platform, and considering some extra space to accommodate the hopping height. The overall height in the frame is about $1.9 \mathrm{~m}$. In the first phase optics aperture and camera parameters are adjusted to have a good image quality. In such conditions the video system is calibrated using a vertical reference with marks every $100 \mathrm{~mm}$. The reference covers the middle part of the image for a height of about $1.2 \mathrm{~m}$. The reference is located at the same distance from the camera of the instrumented right hand side of the subject.

The image is processed with a MatLab ${ }^{\circledR}$ script to identify the ticks and a linear regression is carried out to obtain video system sensitivity. Results are pretty stable and reproducible: 
generally speaking, after linear regression on 12 references (about $1.2 \mathrm{~m}$ ) we obtained a sensitivity of about $2.9 \mathrm{~mm} /$ pixel, with a standard deviation of residuals less than 0.3 pixels and a very good linear behaviour along the image height. Reproducibility due to different measurement setup is very good, since sensitivity variations are usually less than $0.2 \%$. Thanks to the high quality of the optics and camera CCD sensor, a more detailed calibration procedure, following for example DLT or Zhang methods [26]-[27], has shown both negligible distortion correction and negligible anisotropies for this application.

Besides that, the calibration image gives the reference point for the zeroing of video readings in both vertical and sagittal direction, so that the video results will be strictly related to the force platform readings as schematised in Figure 2. For this purpose, the calibration reference is positioned in the horizontal direction on the force platform left edge: from a calibration image it is possible to measure video CoP zero position obtaining the common point between video and force sensors.

\subsection{IMU}

IMU sensors require an initial set up procedure. They are placed horizontally on the floor, with local $\mathrm{Z}$ axis aligned along the frontal axis near the test site; after activation, they rest quiet for at least one minute to warm up and stabilise the readings, and then readings are zeroed in this position. A static recording of the four sensors is carried out before installing them on the subject.

\subsection{Subject set up}

As discussed in Section 3, the test case requires the detailed measurement of CoM together with leg and foot movements. For this reason seven anatomical landmarks are selected for marker placement, as presented in Table 4. They allow the measurement of both absolute and relative angles at the joints, for the four 'rigid' segments considered: foot, lower leg, thigh and torso with arms and head. The same segments are instrumented with IMU sensors [12], [15], [21].

A running suit with a full set of markers sewn in proper position is available, but in order to obtain a more accurate marker positioning on each subject, we found that sticking of each marker on the skin is far better and it avoids suit

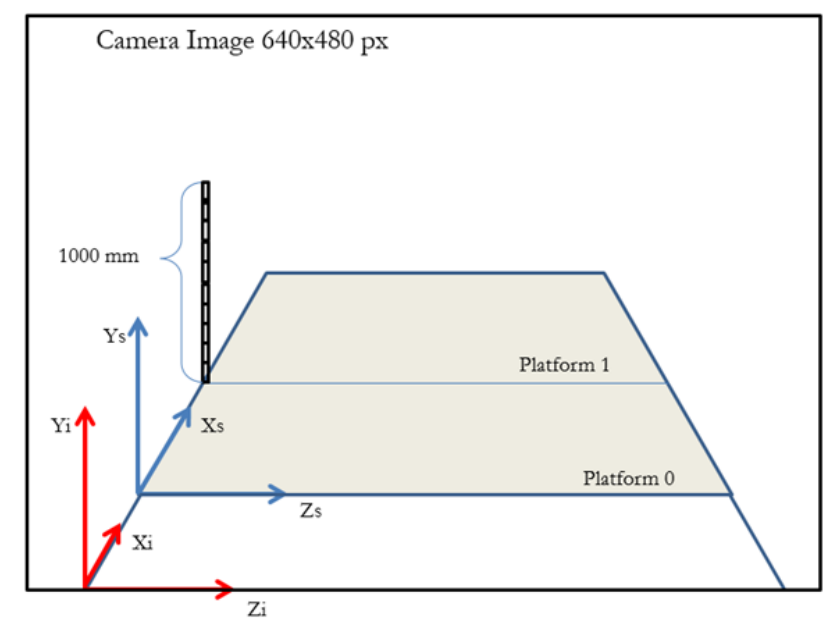

Figure 2. Calibration setup. $Z_{i}$ is the front to rear direction on the image plane; $Z_{s}$ is the same direction but measured by force platforms. The common origin is given by the calibration image.
Table 4. Markers and IMU positions.

\begin{tabular}{cccc}
\hline IMU position & IMU number & Marker position & $\begin{array}{c}\text { Marker led } \\
\text { number }\end{array}$ \\
\hline \multirow{2}{*}{ Foot back } & 1 & Foot tip & 1 \\
& & Metatarsus & 2 \\
Tibiae & 2 & Heel & 3 \\
& & Ankle & 4 \\
Femur & 3 & Knee & 5 \\
Lower back & 4 & Hip & 6 \\
\end{tabular}

movements on the skin.

Once markers are placed on subject's right side facing the camera, IMU sensors are placed on each segment by using Velcro elastic straps.

Force platforms are zeroed and a static acquisition of subject standing quiet is carried out before proceeding with hopping tests.

\subsection{Tests}

Once subject is properly instrumented, force platforms are zeroed and camera parameters are set to obtain black images with white markers. We are ready now for a test session.

The hopping test is performed with a reference sound giving $2.2 \mathrm{~Hz}$ pace. After a short training, each subject is able to reproduce the hopping pace: test starts when the subject feels a good accordance with the pace, and it lasts for at least 10-15 s (about 20-30 events). Then the subject rests for some time, while data are checked. An experimental session consists of several tests performed both naturally, i.e., without taking care of height performance except for pace, and forcing the hopping to obtain a higher height, while maintaining the pace [24]. Test session ends, as it was started, with a static recording. After the test, all the measurement data are verified and if they present inconsistencies, such as misalignments in still measurements, rhythm anomalies and so on, the test is discarded.

\subsection{Data processing}

The processing requires some subsystem specific preprocessing to obtain properly synchronised and scaled data. Video files are processed in Lab-View ${ }^{\circledR}$, identifying the positions of white marker centres in the dark image and then verifying marker tracking by vertical alignment from foot to shoulder [28]. Marker positions are saved in a file for further processing. IMU signals are processed by Xsens software package, MTw Manager, to obtain angle data. Force platform and COP data are exported as separate files for further processing.

Further processing is carried out in Matlab $\AA$ and consist of: - Synchronisation of signals from the three subsystems considering in particular the IMU measurement trigger.

- Resampling of all the data at $200 \mathrm{~Hz}$, to obtain a common time reference.

- Restitution of markers position in millimetres, by considering sensitivity obtained during calibration.

- Evaluation of segment absolute and relative angles from video and IMU sensors.

- Determination of the ground and aerial hopping phases by using a threshold on the vertical force signal.

- Evaluation of quantities at touch down and their excursions at ground.

- Data verification.

- Data presentation and output. 


\section{EXPERIMENTAL RESULTS}

The focus of our experimentation is to validate the proposed measurement system. For this purpose we present here some results obtained from three male subjects (24-50 years old, 1.70$1.80 \mathrm{~m}, 64-70 \mathrm{~kg}$ ) hopping at natural and maximum height. To validate the proposed measurement systems we compare quantitative results from one subject, with data available in literature, and we propose self-consistency tests and an evaluation of the measurement uncertainty.

Figures 3 and 4 present time histories of vertical ground reaction force $(\mathrm{GRF})$ and of the markers position. In Figure 3 it is possible to identify hopping ground and aerial phases, as obtained by thresholding the GRF. Note that the force is normalised according to subject weight, in order to be able to compare different subjects. In Figure 4 it is possible to appreciate hopping repeatability and the small movement of the subject in the ante-posterior direction during the performance.

Figure 5 presents joints angles together with ground phase indication: again good repeatability is evident.

It is possible to make a comparison with literature values considering the ground phase. We can consider values for some

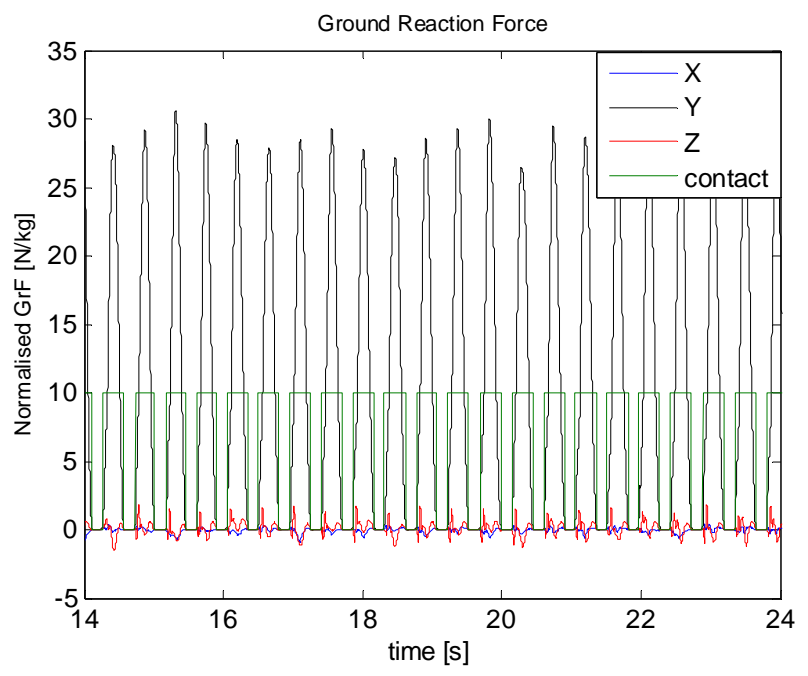

Figure 3. Ground reaction force components and contact signal as measured by force platform and normalized on subject weight.

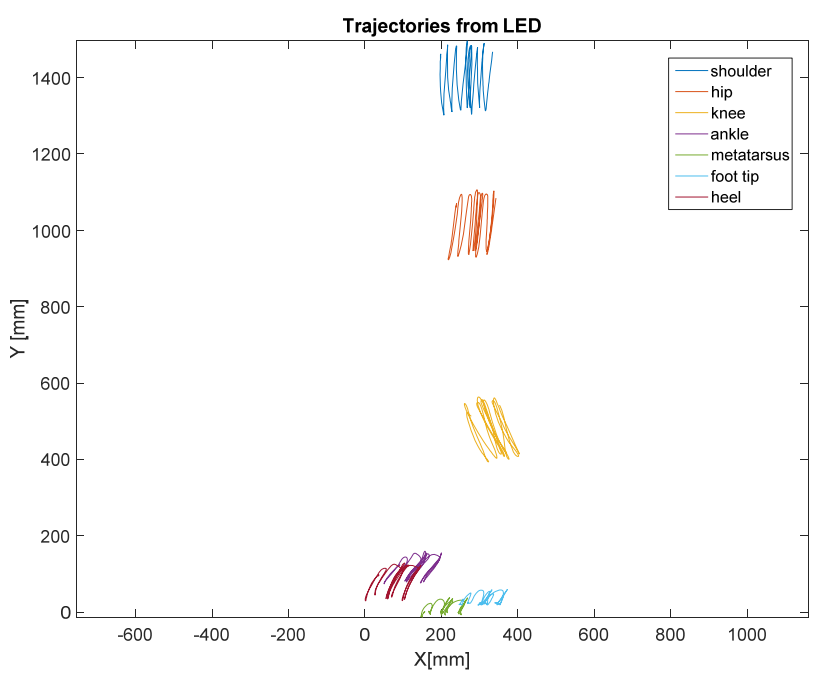

Figure 4. Marker trajectories in the sagittal plane.

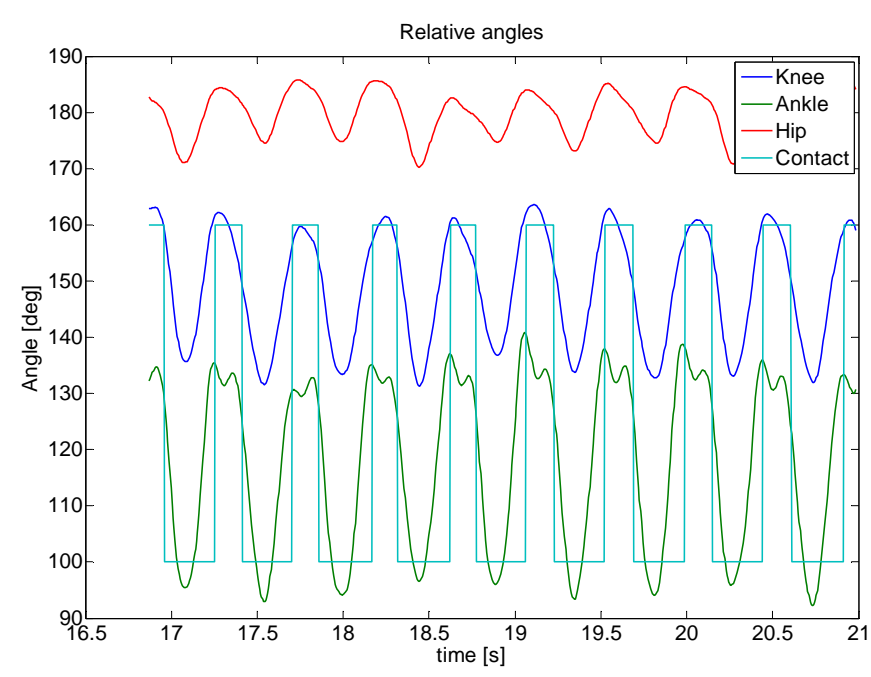

Figure 5. Relative angles at lower limb joints obtained by marker positions measured by the video system

quantities of interest at touchdown and their variations during the ground phase, as presented in Table 5 for a $65 \mathrm{~kg}, 1.72 \mathrm{~m}$, male subject, together with some reference values from the literature [13].

Mean values show a rather good agreement with literature considering that results from this study refers to repeated executions of the test by a specific subject while in literature a rather large set of subjects was considered, with balanced gender and age composition. We are instead mainly interested in validating the procedure, rather than in obtaining statistics for a population of subjects, and we therefore focus on a single subject.

However it is worth noting that absolute angles at touchdown depend on initial marker alignment on the subject's limb. This is particularly critical since it requires very accurate marker sticking on anatomical landmarks. If some of them can be identified on the skin surface with good accuracy, (malleolus, knee) for others this is rather difficult (metatarsus, hip and shoulder in alignment with hip). This results in a bias in touchdown angle evaluation and systematic uncertainties in relative ones, as will be discussed in Section 5.3. This is evident from values in Table 5, where it appears that the hip angle at touchdown and relative angle at the ankle presenting the largest deviation from literature.

Table 5. Leg quantities during ground phase. Literature reference values from [13].

\begin{tabular}{ccccc}
\hline Quantity & Experiment & $\boldsymbol{\sigma}$ & Literature & $\boldsymbol{\sigma}$ \\
\hline Hopping rate $[\mathrm{Hz}]$ & 2.2 & $1.8 \%$ & & \\
Time spent at ground $[\mathrm{ms}]$ & 297 & 7.5 & 308 & 8 \\
Ground reaction force $[\mathrm{N}]$ & 1860 & 103 & 1740 & 87 \\
\hline Touchdown angles [deg] & & & & \\
\hline Hip & 180 & 1.5 & 171 & 3.7 \\
Knee & 154 & 3 & 150 & 4.81 \\
Ankle & 126 & 1.9 & 125 & 3.09 \\
\hline Excursion at ground [deg] & & & & \\
\hline Hip & 12 & 1.9 & 10 & 1.3 \\
Knee & 26 & 1.7 & 23 & 2.3 \\
Ankle & 42 & 4.2 & 32 & 1.0 \\
\hline
\end{tabular}




\subsection{Details on the ground phase}

Moving now to another aspect of this study, we consider the displacements of whole body centre of mass (CoM) and of centre of pressure (CoP). CoP and $\mathrm{CoM}$ positions may be measured directly through the markers data or evaluated considering the CoM of each body segment and its position in the space, together with anthropometric data. This second procedure may use of marker video data, or orientation data from the inertial sensors. Figure 6 presents a time history for the frontal movement in the sagittal plane of hip and metatarsus markers, CoP and the whole body CoM. The ground phase is evident since no CoP data are available during flight. Figure 7 presents a detail for a single ground phase.

During the ground phase we can consider that, since the CoM is located just above the hip marker, and the torso is a bit inclined and not fully upright, the CoM position is a bit displaced ahead from the hip. From a dynamical point of view the CoP at touchdown tends to the back and returns in the front of the foot, while the CoM presents a smoother behaviour in accordance with CoP.

In addition to the points previously described, the instantaneous centre of rotation (CoR) plays an important role for subject stability. Generally it is assumed to be coincident

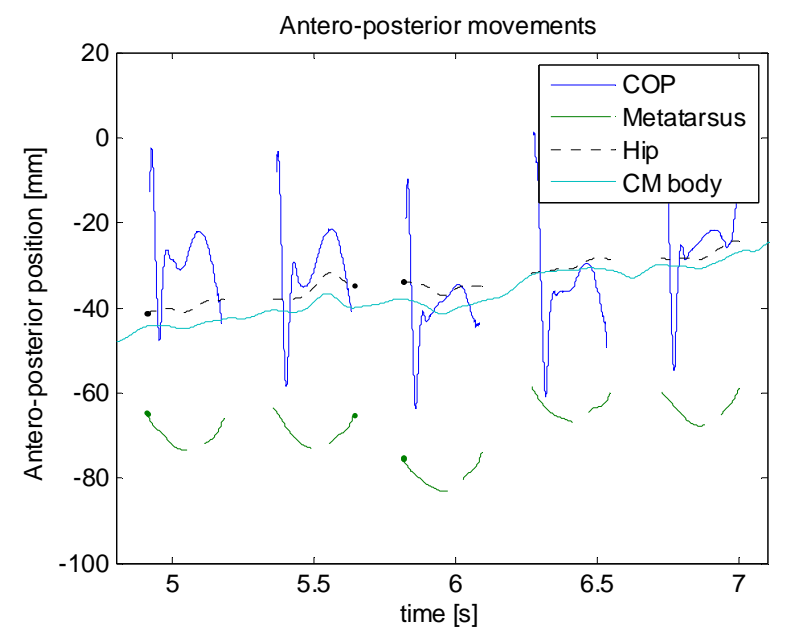

Figure 6. Antero-posterior movements of CoP, CoM as measured at the hip, CoM evaluated from segments positions and metatarsus considered as foot rotation center

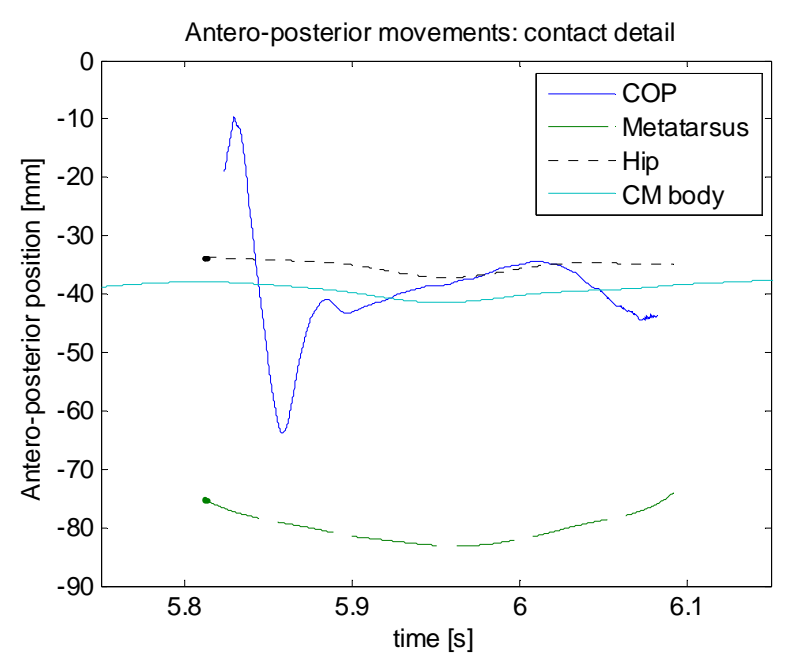

Figure 7. Detail of anterior-posterior ground reaction force and COP position during a single ground phase. with foot metacarpus, identified by a specific marker, yet it might be interesting to measure its position. A possibility is to assume the foot top as a rigid surface and considering velocity vectors measured at malleolus and metacarpus markers. Since a foot top can be considered as a rigid element, at every instant the two straight lines by the extremes of the rigid element, corresponding to the velocity vectors, intersect in the instantaneous centre of rotation. Such procedure presented some computational criticalities probably due to the composition of rotational and translational movements, so for the moment we will take the metacarpus position as foot centre of rotation. As shown in Figure 6, CoR, or metatarsus, during the ground phase moves from an anterior position backward and then returns ahead before take-off, following the movement of the foot.

It is possible to analyse anterior-posterior force also, as presented in Figure 8. Here anterior-posterior force component follows the CoP movement to maintain equilibrium during contact and hopping stability as shown for a set of successive hopping presenting some anterior posterior CoM movement which is compensated by the force component in the same direction.

As regards left to right force component, this is not of interest in this case since we are not studying gesture symmetry, but we assume the same behaviour for the two legs. This approach seems to be reasonable for a healthy subject hopping in a stable position as shown in Figure 9.

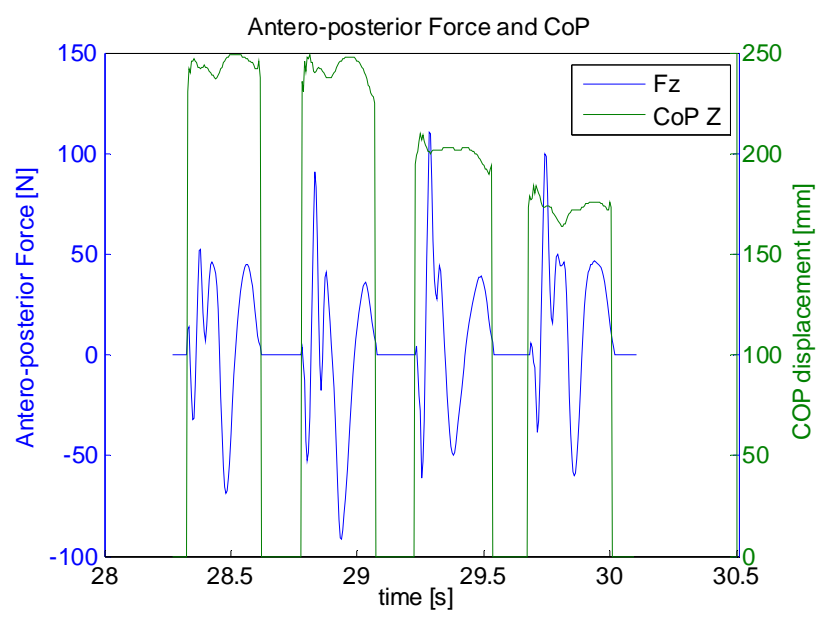

Figure 8. Antero-posterior ground reaction force and COP position

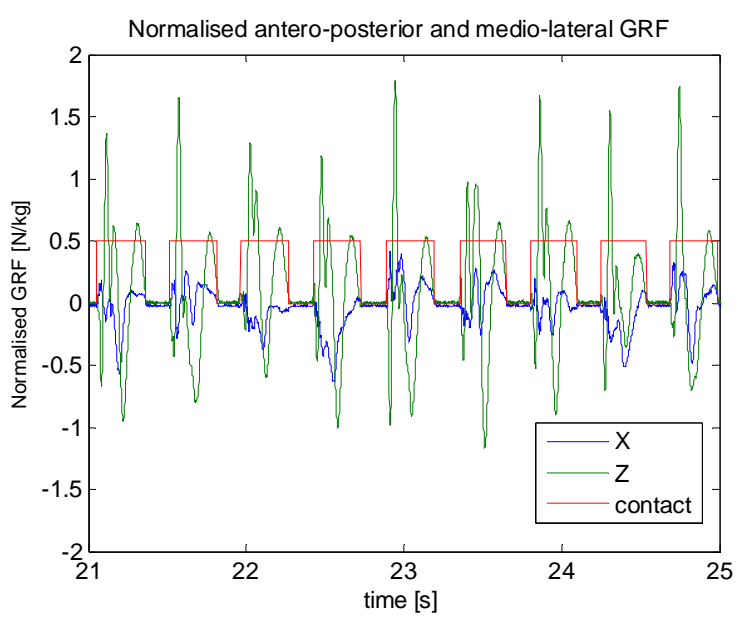

Figure 9. Normalised antero-posterior and medio-lateral GRF components. 


\subsection{Data verification}

As discussed in Section 2, the present measurement system architecture offers several data verification possibilities. We can introduce for example angle measurements from video and IMU systems, as presented in the following Figure 10. Angles from the video system are computed by trigonometric formulae applied to marker positions measurements, while the IMU system measures angles directly [23]. In general we obtained a good agreement between the two measurement systems: the mean differences between the angles measured at -ankle, -knee and -hip are respectively 1.7, 1.9 and 1.6 degrees with a relative standard deviation about $0.81,3.7$, and $4.3 \%$.

As an example we can consider the hip angle. Figure 10 shows the limited differences between the two independent measurement methods. Nevertheless it is worth noting that small differences might be due to a misalignment between the sagittal plane, assumed coincident with the plane of the image, and the IMU coordinate system, due for example to IMU sensor placement or to its movement during hopping. For this reason this effect is different on each angle and it varies from a minimum of $\pm 3 \mathrm{deg}$ up to $\pm 5 \mathrm{deg}$ for the foot, when the IMU is near the force platform.

Another verification possibility regards the length of body segments, which we are assuming as rigid. Figure 11 presents

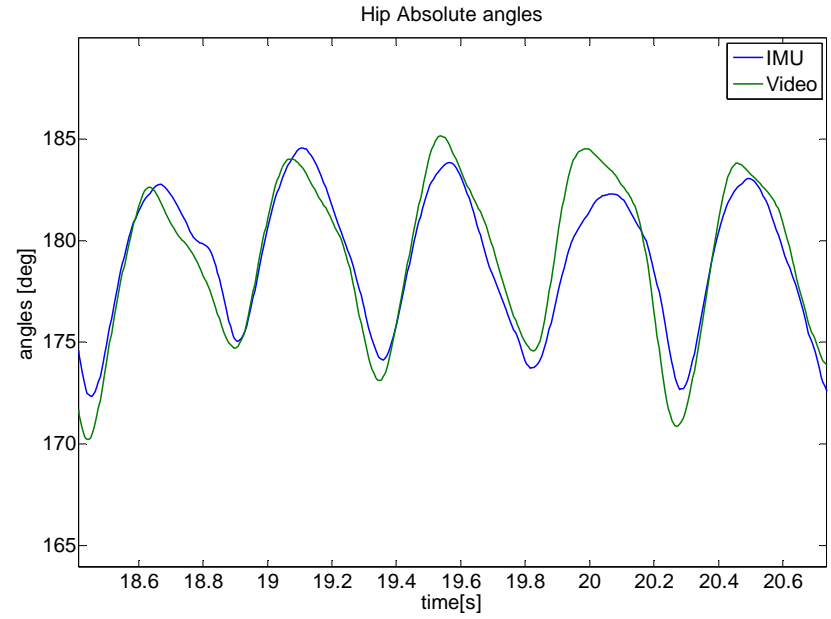

Figure 10. Hip angle measurements verification: comparison of the readings obtained by markers plus video and by IMU.

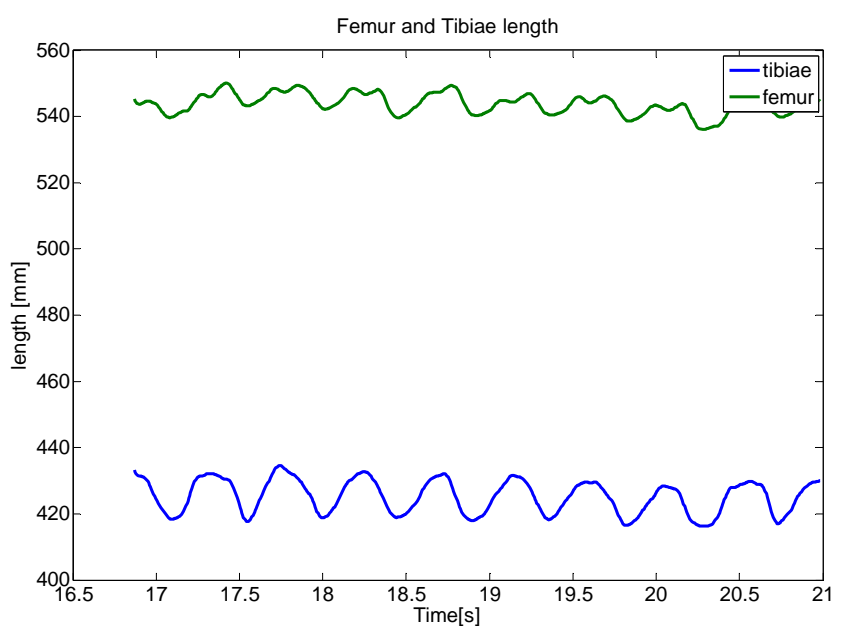

Figure 11. Validation of marker position measurement by video: femur and tibiae lengths the femur and tibiae lengths as a function of time during the hopping. Segments lengths are evaluated considering the markers distance between malleolus and knee (tibiae) and between knee and hip (femur). They are not rigorously constant, probably because of both small marker misplacements from the expected landmark, and of markers movement from their reference positions during performance. Since markers are stitched on the skin to avoid suit movements, the effect is probably due to skin itself that moves during hopping performance in respect to segment rigid frame or bone. This is a typical problem in biomechanics [6], but in this case we had the possibility to directly quantify these effects. Both the rather small variation in segment length, which is less than $1.5 \%$ for tibiae, the worst case, and the good agreement on the angles computed from video and inertial data, give confidence about measurement system reliability.

\subsection{Uncertainty evaluation}

Let us now discuss the main uncertainty sources, as identified in the experimentation here presented. Systematic uncertainty contributions are mainly due to misalignment of the video measuring system and IMU with subject. Another important factor is marker placement [2], [29]. Angular misalignments produce a second order effect that is negligible if the camera is properly oriented during setup. In fact subject's rotation around the vertical axis during hopping are not common and in general they require test interruption to recover equilibrium. Through geometrical considerations it is possible to estimate the effect of such a rotation on position and angle measurements. For a rotation around vertical axes of $5 \mathrm{deg}$, which is the maximum we expect during performance, we obtained a $0.7 \%$ deviation on all position measurements. It has no effect on angle measurements, since they are based on relative positions.

Angle measurements might be affected, as already introduced in Section 5.2, by marker positioning. We can distinguish two situations when dealing with absolute or relative angles. For absolute angles we can distinguish between markers moving or misplaced along the segment or we can hypothesize movements along the camera horizontal and vertical axis. In the former case it produces no effect. In the latter, it is possible to evaluate a deviation of about $1 \mathrm{deg}$ for the experienced length variations. For relative angles we have to consider the correlation between marker movements. Let's focus on knee angle and markers of tibiae and femur. In the case presented in Figure 11, we have a linear correlation coefficient of about 0.8 , indicating that most probably the knee marker was placed a little bit forward in respect to the knee centre of rotation. A geometrical evaluation of the corresponding knee angle mean deviation is lower than $1 \mathrm{deg}$, variable with the angle itself. Of course other marker movements might contribute, but the very limited segment length variation and the good correspondence between video and IMU angles, indicate a proper marker and IMU placement. Nevertheless, ankle angle presents a nearly constant difference, for video and IMU measurements, with a systematic reduction for ankle angle video measurement, as compared with IMU. These phenomena might be explained geometrically, considering metatarsus marker and its placement. If the position of this marker is slightly moved toward foot tip, this will introduce a corresponding reduction in ankle angle measurements. It is possible to estimate this effect geometrically, obtaining, for a displacement of about $3 \mathrm{~cm}$, an 
angle measurement reduction of about $20 \%$, that corresponds to the difference experimentally verified between video and IMU. Such a large marker displacement is realistic if we consider that estimating metacarpus position form foot skin or from outside a shoe is not easy in general, and in particular for non-physician personnel.

Another possible source of uncertainty is subject to camera distance. Deviations of this distance from the standard calibration distance affect video sensitivity and consequently marker position measurements. During tests subject position is independently monitored by the CoP readings, so that it is possible to evaluate this uncertainty contribution experimentally. Distance variations are less than $10 \%$ and the same relative contribution affects marker position measurements. Note that such effect does not contribute to angle uncertainty since it determines a proportional horizontal and vertical variation, without affecting angles.

To sum up, if a proper set up of the system is carried out, main uncertainty contributions are due to marker placement, which might heavily affect measurements. On the other hand data verification with the IMU sensor eventually makes this problem evident, giving the opportunity to compensate data or to correct marker position on the subject.

As regards random contributions of course they are dominated by repeatability during a repeated task, due to subject ability to keep the correct pace, and by reproducibility when considering different subjects or test sessions carried out during different days.

\section{CONCLUSIONS}

The measurement of human movement has been considered in a metrological perspective. A complex and partially redundant measurement system has been developed and thoroughly tested. The system will be used, in the future, for studying various gestures, including on site high jump or spinning, walking and running. As validation test case we have considered hopping since it presents several measurement issues and measurement data are available in literature. Furthermore we think that in order to understand properly hopping dynamics, a better characterisation of the contact conditions is required, especially if stability issues have to be studied.

In the approach here presented, a combination of marker, video and inertial sensors enable results verification and internal validation. Validation and verification methods are presented in the paper together with uncertainty evaluation and a discussion on main uncertainty contributions and their control. The main issue in this kind of measurement is connected to marker positioning. The proposed approach gives the operator the possibility to verify results, pointing out experimental tests, potential problems and successively enables measurement uncertainty evaluation. IMU sensors demonstrate high flexibility and simple setup on the subject; they are a good choice in combination with video systems, even if they still present a rather large uncertainty due to the effect of the surrounding magnetic environment.

\section{ACKNOWLEDGEMENT}

This research activity has been partially funded through University of Genova Research Projects PRA 2013-2014.

\section{REFERENCES}

[1] Losier K H, Eriksson A, Leg stiffness measures depend on computational method, J. Biomechanics, 47 (2014), pp. 115-121.

[2] Maletsky LP, Sun J, Morton NA, Accuracy of an optical active marker system to track the relative motion of rigid bodies, J. Biomechanics, 40 (2007), pp. 682-685.

[3] Hendrick TL, Software techniques for two and three dimensional kinematic measurements of biological and biomimetic systems, Bioinspiration and Biomimetics 3 (2008), doi: 10.1088/17483182/3/3/034001.

[4] Winter DA, Kinematic and kinetic patterns in human gait variability and compensating effects, Human Movement Science 3 (1984), pp. 51-76, North Holland

[5] Favre J, Crevoisier X, Jolles BM, Evaluation of a mixed approach combining stationary and wearable systems to monitor gait over long distance, J. Biomechanics, 43 (2010), pp. 2196-2202.

[6] Cappello A, CappozzoA, Della Croce U, Leardini A, "Bone position and orientation reconstruction using external markers" in Three dimensional analysis of human locomotion. Wiley, New York 1997, pp. 147-172.

[7] Winter D.A., Biomechanics and Motor Control of Human Movement, Wiley, New York, 4th edition 2009.

[8] Hobara H, Kimura K, Omuro K, Gomi K, Muraoka T, Iso S, Kanosue K, Determinants of difference in leg stiffness between endurance- and power-trained athletes, J. Biomechanics, 41 (2008), pp. 506-514.

[9] Nigg B.M., Herzog W., "Biomechanics of the Musculo-skeletal System”, Wiley, New York, 3rd edition 2007.

[10] Crenna F, Rossi GB, Kinematic measurements for the characterization of the dynamics of hopping, Journal of Physics: Conference Series 588, (2015) doi:10.1088/1742-6596/588/1/012044

[11] Picierno P, Cereatti A, Capozzo A. Joint kinematic estimate using wearable inertial and magnetic sensing modules. Gait and Posture, Volume 28 (2008), pp. 588-595.

[12] Zhou H, Stone T, Hu H, Harris N., Use of multiple wearable inertial sensors in upper limb motion tracking. Medical Eng. \&Physics, 30, (2008), pp. 123-133.

[13] Farley C T, Morgenroth D C, Leg stiffness primarily depends on ankle stiffness during human hopping, J. Biomechanics 32 (1999), pp. 267-273.

[14] Rapoport S, Mizrahi J, Kimmel E, Verbitsky O, Isakov E, Constant and variable stiffness and damping of the leg joints in human hopping. J. Biomechanical Engineering, 125 (2003), pp. 507-514.

[15] Findlowa, J.Y. Goulermas, C. Nester, Predicting lower limb joint kinematics using wearable motion sensors, Gait \& Posture 28 (2008), pp. 120-126.

[16] S. Kuitunen, K. Ogiso and P. V. Komi, Leg and joint stiffness in human hopping, Scandinavian Journal of Med. \& Sci. in Sports 21, 6 (2011), pp. 159-167.

[17] Hobara H., Inoue K., Muraoka T., Omuro K., Sakamoto M., Kanosue K., Leg stiffness adjustment for a range of hopping frequencies in humans, J. Biomechanics. 43 (2010), pp. 506-511.

[18] Farley CT, Houdijk HHP, Van Strien C, Louie M., Mechanism of leg stiffness adjustment for hopping on surfaces of different stiffness, J Appl Physiol. 85 (1998), pp. 1044-1055.

[19] Liu T, Inoue Y, Shibata K, Development of a wearable sensor system for quantitative gait analysis, Measurement 42 (2009), pp. 978-988.

[20] Farley C T, Blickhan R, Saito J, Taylor C R, Hopping frequency in humans: a test of how springs set stride frequency in bouncing gaits, J.Applied Physiology 71 (1991), pp. 2127-2132. 
[21] Spaepen A.J., Peeraer L., Willems E.J., Centre of gravity and centre of pressure in stabilometric studies, Agressologie 20 (1979), pp. 117-118

[22] Moritz C T, Farley C T, Human hoppers compensate for simultaneous changes in surface compression and damping, $\mathrm{J}$. Biomechanics, 39 (2006), pp. 1030-1038.

[23] Masci I, Vannozzi G, Getchell N, Cappozzo A., Assessing hopping developmental level in childhood using wearable inertial sensor devices, Motor Control, 2012 Jul; 16(3), pp. 317-328.

[24] Riese S, Seyfarth A, Grimmer S, Linear center-of-mass dynamics emerge from non-linear leg-spring properties in human hopping. J. Biomechanics, 46 (2013), pp. 2207-2212.

[25] Hobara H, Muraoka T, Omuro K, Gomi K, Sakamoto M, Inoue $\mathrm{K}$, Kanosue $\mathrm{K}$, Knee stiffness is a major determinant of leg stiffness during maximal hopping, J. Biomechanics. 42 (2009), pp. 1768-1771.

[26] B Yu, TJ Koh, JG Hay, A panning DLT procedure for three dimensional videography, J. Biomechanics 26 (1993), pp. 741751.

[27] Z Zhang, A flexible new technique for camera calibration, IEEE Trans. Pattern Analysis and Machine Intelligence 22, 11 (2000), pp. 1330-1334.

[28] F. Bobick, A.D. Wilson, A State-Based Approach to the Representation and Recognition of Gesture, Trans. Pattern Analysis and Machine Intelligence 19, 12 (1997), pp. 1325-1337.

[29] G.B. Rossi, "Measurement and probability", Springer, Dordrecht, (2014), ISBN 978-94-017-8825-0. 